\title{
Detection of Strawberry Diseases Using a Convolutional Neural Network
}

\author{
Jia-Rong Xiao ${ }^{1}$, Pei-Che Chung ${ }^{2}$, Hung-Yi Wu ${ }^{3}$, Quoc-Hung Phan ${ }^{1}$, Jer-Liang Andrew Yeh ${ }^{4}$ \\ and Max Ti-Kuang Hou ${ }^{1, *}$ \\ 1 Department of Mechanical Engineering, National United University, Miaoli 360001, Taiwan; \\ a645411007@gmail.com (J.-R.X.); qhphan@nuu.edu.tw (Q.-H.P.) \\ 2 Miaoli District Agricultural Research and Extension Station, Miaoli 363201, Taiwan; peiche@mdais.gov.tw \\ 3 Department of Plant Pathology and Microbiology, National Taiwan University, Taipei 106319, Taiwan; \\ hywu0615@gmail.com \\ 4 Department of Power Mechanical Engineering, National Tsing Hwa University, Hsinchu 300044, Taiwan; \\ jayeh@mx.nthu.edu.tw \\ * Correspondence: max@nuu.edu.tw
}

Citation: Xiao, J.-R.; Chung, P.-C.; Wu, H.-Y.; Phan, Q.-H.; Yeh, J.-L.A.; Hou, M.T.-K. Detection of Strawberry Diseases Using a Convolutional Neural Network. Plants 2021, 10, 31. https://dx.doi.org/10.3390/ plants10010031

Received: 30 November 2020 Accepted: 22 December 2020 Published: 25 December 2020

Publisher's Note: MDPI stays neutral with regard to jurisdictional claims in published maps and institutional affiliations.

Copyright: () 2020 by the authors. Licensee MDPI, Basel, Switzerland. This article is an open access article distributed under the terms and conditions of the Creative Commons Attribution (CC BY) license (https: / / creativecommons.org/ licenses/by/4.0/).

\begin{abstract}
The strawberry (Fragaria $\times$ ananassa Duch.) is a high-value crop with an annual cultivated area of $\sim 500$ ha in Taiwan. Over $90 \%$ of strawberry cultivation is in Miaoli County. Unfortunately, various diseases significantly decrease strawberry production. The leaf and fruit disease became an epidemic in 1986. From 2010 to 2016, anthracnose crown rot caused the loss of 30-40\% of seedlings and $\sim 20 \%$ of plants after transplanting. The automation of agriculture and image recognition techniques are indispensable for detecting strawberry diseases. We developed an image recognition technique for the detection of strawberry diseases using a convolutional neural network (CNN) model. CNN is a powerful deep learning approach that has been used to enhance image recognition. In the proposed technique, two different datasets containing the original and feature images are used for detecting the following strawberry diseases-leaf blight, gray mold, and powdery mildew. Specifically, leaf blight may affect the crown, leaf, and fruit and show different symptoms. By using the ResNet50 model with a training period of 20 epochs for 1306 feature images, the proposed CNN model achieves a classification accuracy rate of $100 \%$ for leaf blight cases affecting the crown, leaf, and fruit; $98 \%$ for gray mold cases, and $98 \%$ for powdery mildew cases. In 20 epochs, the accuracy rate of $99.60 \%$ obtained from the feature image dataset was higher than that of $1.53 \%$ obtained from the original one. This proposed model provides a simple, reliable, and cost-effective technique for detecting strawberry diseases.
\end{abstract}

Keywords: strawberry diseases; convolution neural network; image recognition

\section{Introduction}

Crop pests and diseases are major problems in the agricultural industry that cause significant losses to food production. Nearly half of the world's crops are lost due to pest infestation and disease [1]. In Miaoli County, Taiwan, the strawberry, which contributes $\$ 1.8$ billion annually, is facing similar problems. Strawberries have high nutritional content, commercial value, and are a major fruit for daily consumption [2,3]. Strawberries are easily infected by several phytopathogenic fungi, bacteria, and viruses [4-6]. The main strawberry pathogens are as follows-Colletotrichum siamense, which causes anthracnose [7,8]; Botrytis cinereal, the causal agent of gray mold [9,10]; Neopestalotiopsis spp. [11], the causal agent of crown rot, fruit rot and leaf blight [7]; and other fungi that cause powdery mildew, which affects petioles [12], leaves, and fruits in a strawberry-specific manner [13]. These pathogens interfere with photosynthesis and negatively impact fruit quality, growth, and productivity. Strawberry diseases are manually identified by growers, which is laborious and time-consuming. The shrinking workforce in agricultural counties also complicates this issue, since it is harder to accurately predict disease severity over a large 
scale. Developing a rapid, accurate, automated technique to detect strawberry diseases is required.

Recently, deep learning artificial intelligence (AI) technology has been applied for the detection of crop diseases [14,15]. Machine learning (ML) image recognition has been used as a highly accurate and inexpensive tool for detecting crop diseases [16,17]. In published reports, the convolution neural network $(\mathrm{CNN})$ is one of the most popular ML techniques for detecting crop diseases. Jeon and Rhee [18] proposed the CNN technique for plant leaf recognition using the GoogLeNet model. The proposed technique was able to detect damaged leaves with a recognition rate of $>94 \%$, even when only $30 \%$ of the leaf was damaged. Mohanty et al. [19] used CNN for detecting crop species and diseases based on a public dataset of images using GoogLeNet and AlexNet training models. Based on the color, grayscale, and leaf segmentation, the proposed model was $99.35 \%$ accurate. Selvaraj et al. [20] proposed deep transfer learning (DTL) for the detection of banana diseases and pest an the accuracy of $90 \%$. Many deep learning AI techniques have been proposed for predicting strawberry quality. Sustika et al. [21] used five different types of deep CNN architectures, including AlexNet, MobileNet, GoogLeNet, VGGNet, and Xception for inspecting strawberry quality. These proposed techniques may be up to $95 \%$ accurate. Shin et al. [22] used supervised ML technologies to detect strawberry powdery mildew disease with an accuracy of $94.34 \%$. These studies in [22-24] have provided a foundation for AI-mediated disease detection. However, CNN has not yet been used for strawberry disease detection. In this study, we used CNN techniques and a ResNet50 model to detect leaf blight (which infects the crown, leaf, and fruit), gray mold, and powdery mildew from the "Taoyuan No. 1" and "Xiang-Shui" strawberry cultivars in Miaoli County, Taiwan. The objective of this study is to apply the state-of-the-art CNN techniques for the detection of leaf blight, gray mold, and powdery mildew from the "Taoyuan No. 1" and "Xiang-Shui" strawberry cultivars in Miaoli County, Taiwan. We also compare the accuracy of results obtained from three different models namely VGG-16, GoogLeNet, and Resnet-50 to confirm the feasibility of the proposed technique for strawberry disease detection.

\section{Results and Discussion}

\subsection{GoogLeNet Model of the Confusion Matrix}

The confusion matrix shows the final detection results of the original and feature image dataset with the training period of 20 epochs of the GoogLeNet model (Figure 1). The diagonal axis number presents the correct number of classifications and the rest presented are misclassifications. The classification accuracy rate was $100 \%$ for leaf blight caused by crown rot and powdery mildew for the original image dataset (Figure 1a). The classification rate was $84 \%$ with 26 correct images for gray mold, $84 \%$ with 26 correct images for leaf blight affecting the fruit, and $90 \%$ with 28 correct images for leaf blight. For the feature image dataset, the classification accuracy rate was $100 \%$ for powdery mildew, leaf blight, and fruit rot (Figure 1b). The classification rate was $96 \%$ with 48 correct images for gray mold and $96 \%$ with 48 correct images for crown rot caused by leaf blight.

\subsection{VGG16 Model of the Confusion Matrix}

Figure 2 shows the confusion matrix of the final detection results of the original and feature image dataset with the training period of 20 epochs using the Vgg16 model. The diagonal axis number presents the correct number of classifications and the rest presented are misclassifications. For the original images dataset, the classification accuracy rate is $100 \%$ for leaf blight caused crown rot and powdery mildew (Figure 2a). The classification rate was $97 \%$ with 30 correct images for gray mold, $84 \%$ with 26 correct images for leaf blight affecting fruit, and $90 \%$ with 28 correct images for leaf blight. For the feature image dataset, the classification accuracy rate was 100\% for crown rot caused by leaf blight, leaf blight, fruit rot, and gray mold as well as $96 \%$ with 48 correct images for powdery mildew (Figure 2b). 


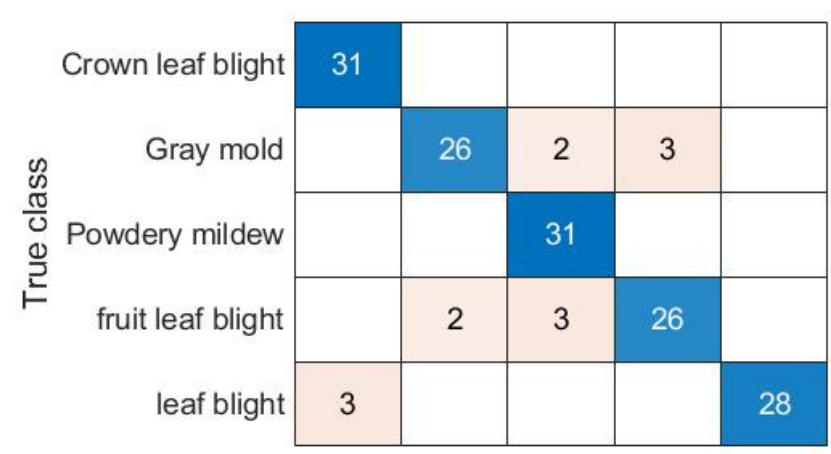

(a)

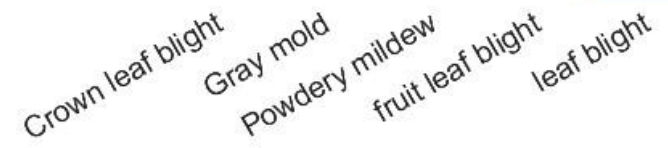

Predicted class

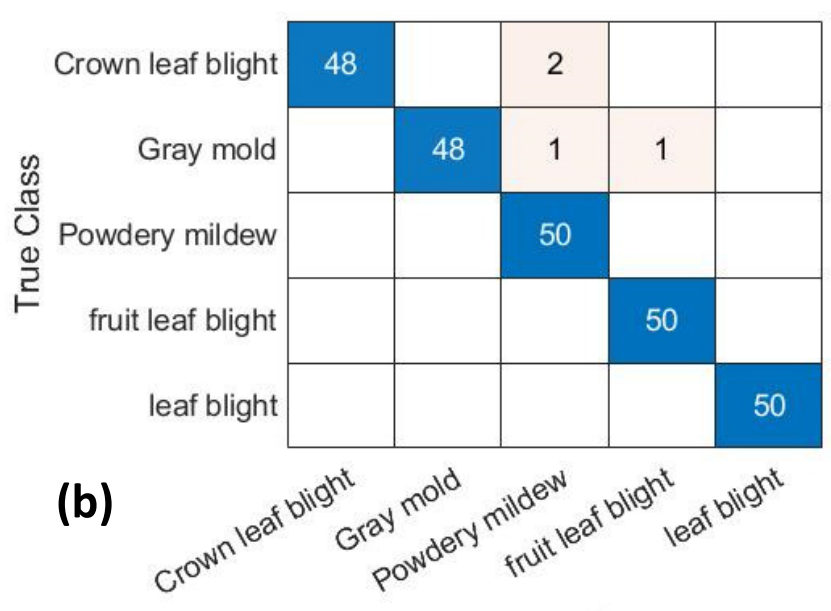

Predicted Class

Figure 1. Confusion matrix with the GoogLeNet model of 20 epochs of the (a) original dataset, and (b) feature dataset.

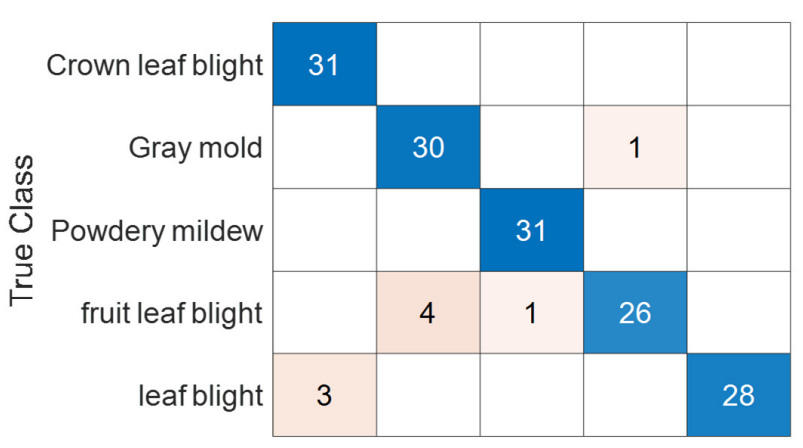

(a)

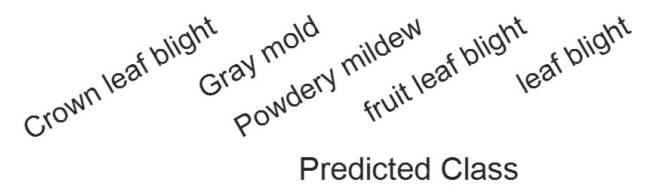

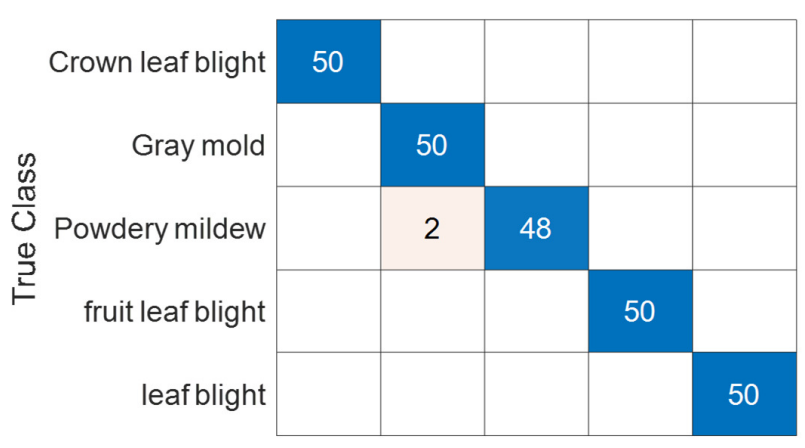

(b)

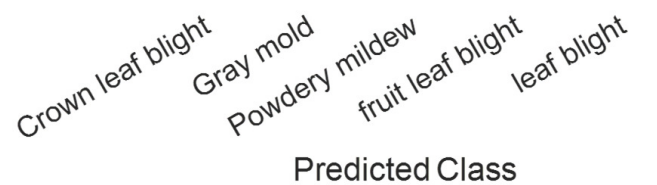

Figure 2. Confusion matrix with the VGG16 model of 20 epochs of the (a) original dataset, and (b) feature dataset.

\subsection{Resnet50 Model of the Confusion Matrix}

Figure 3 shows the confusion matrix of the final detection results of the original and feature image datasets with a training period of 20 epochs for the Resnet50 model. The diagonal axis number presents the correct number of classifications and the rest presented are misclassifications. For the original images dataset, the classification accuracy rate was $100 \%$ for leaf blight-induced crown rot and leaf blight (Figure 3a). The classification rate was $97 \%$ with 30 correct images of gray mold, $84 \%$ with 26 correct images of leaf blight-induced fruit rot, and 90\% with 28 correct images for leaf blight. For the feature image dataset, the classification accuracy rate was $100 \%$ for leaf blight-induced crown rot, leaf blight, and fruit rot (Figure $3 \mathrm{~b}$ ). The classification rate was $98 \%$ with 49 correct images for gray mold and $98 \%$ with 49 correct images for powdery mildew. The results also showed that for two types of leaf diseases, the system uses clear leaf patterns in the original and feature image datasets to detect without error. For the leaf blight-induced fruit rot, the classification rate increases to $100 \%$ for the feature image dataset. Thus, the trimmed image can minimize the complexity pattern and increase the accuracy of classification. Similarly, the classification rate is also improved for leaf blight causing fruit rot type from $84 \%$ by the original image dataset to $99.99 \%$ by the feature image dataset. The lower performance of the original image dataset can be explained by the complexity of the background pattern leading to the confusion of the system. Notably, this classification rate is comparable 
with that of the faster R-CNN multi-task learning proposed in $[25,26]$ and higher than that of the supervised machine learning in $[21,24]$. In general, the proposed technique provides a potential method for five major strawberry diseases' detection. The classification rates obtained from the feature image dataset are better than those obtained from the original image dataset with all three models. The fruit leaf blight and leaf blight diseases are detected with $100 \%$ classification rate with all three models. Furthermore, the crown leaf blight disease is detected with a $100 \%$ classification rate with only two models of VGG16 and Resnet 50 . The gray mold disease is detected with a $100 \%$ classification rate with only the VGG16 model. The powdery disease is detected with a 100\% classification rate with only the GoogLeNet model. In the future, more research will be conducted to compare the accuracy of the results obtained by the proposed technique with those obtained from an experienced crop scout in the field. Furthermore, based on the fruitful results obtained from the proposed technique, a full automated artificial intelligent mobile app will be developed to perform disease detection in the early stage. Thus, it will control the yield losses for commercial Strawberry production in Taiwan and help millions of users in developed countries.
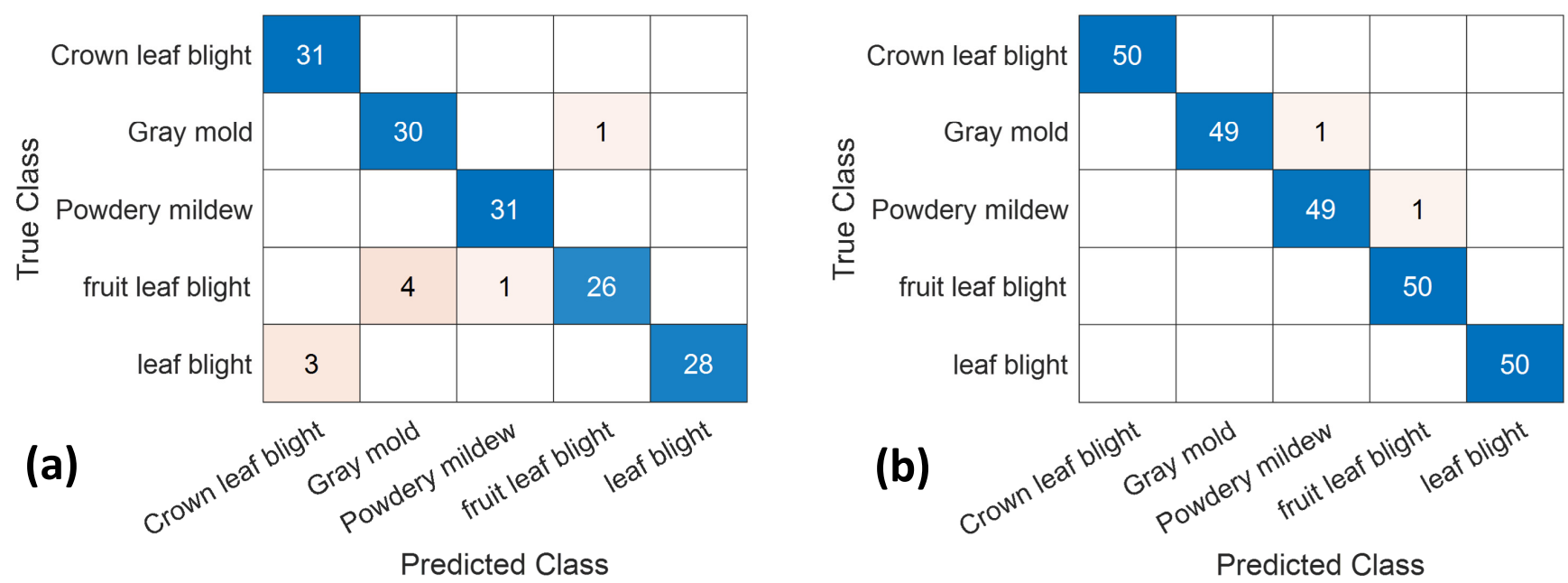

Figure 3. Confusion matrix with the Resnet50 model of 20 epochs of the (a) original dataset, and (b) feature dataset.

\section{Materials and Methods}

\subsection{Strawberry Diseases Dataset}

Images were taken for the "Taoyuan No. 1" and "Xiang-Shui" strawberry cultivars. These images were taken at a strawberry farm at Dahu Township, Miaoli County using a Sony RX10ii camera. During the shooting process, a plant pathologist identified the type of disease and marked them by nametags. Five types of strawberry disease images including leaf blight (crown rot, leaf blight, fruit rot), gray mold, and powdery mildew were collected. We choose these 5 types of disease for detection purpose because they are major diseases caused the yield lost for commercial strawberry production in Taiwan. A total of 792 images were taken and named as original images as shown in Figure 4. One original image can produce several feature images. Feature images were manually trimmed without using any background subtraction methods as shown in Figure 5. Withered areas on the leaves in the leaf blight group minimized the variation of nodule contrast. 

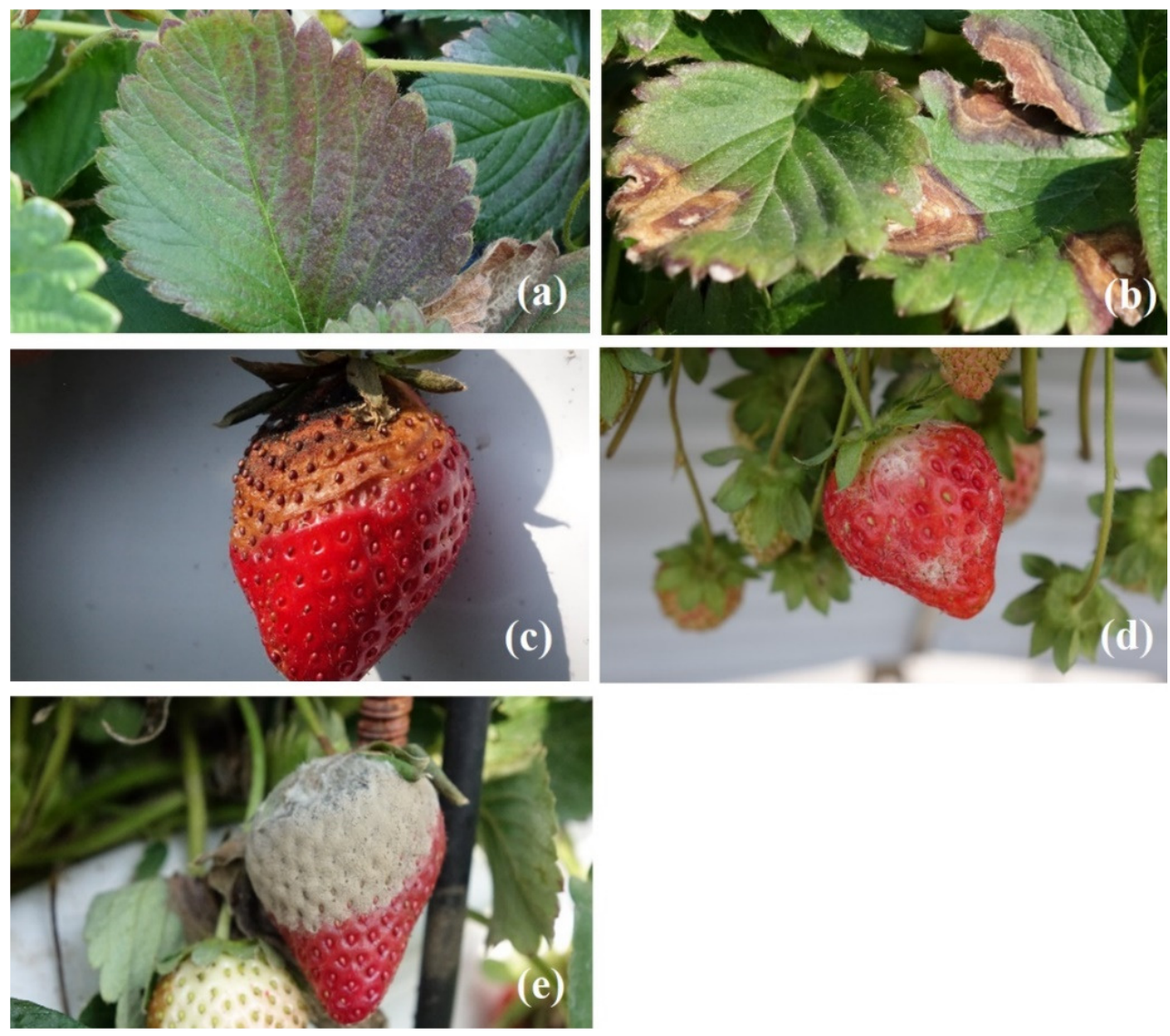

Figure 4. A total of 792 original images for different diseases of strawberry plant(a) crown rotcaused by leaf blight, (b) leaf blight, (c) fruit rot caused by leaf blight, (d) powdery mildew, and (e) gray mold.

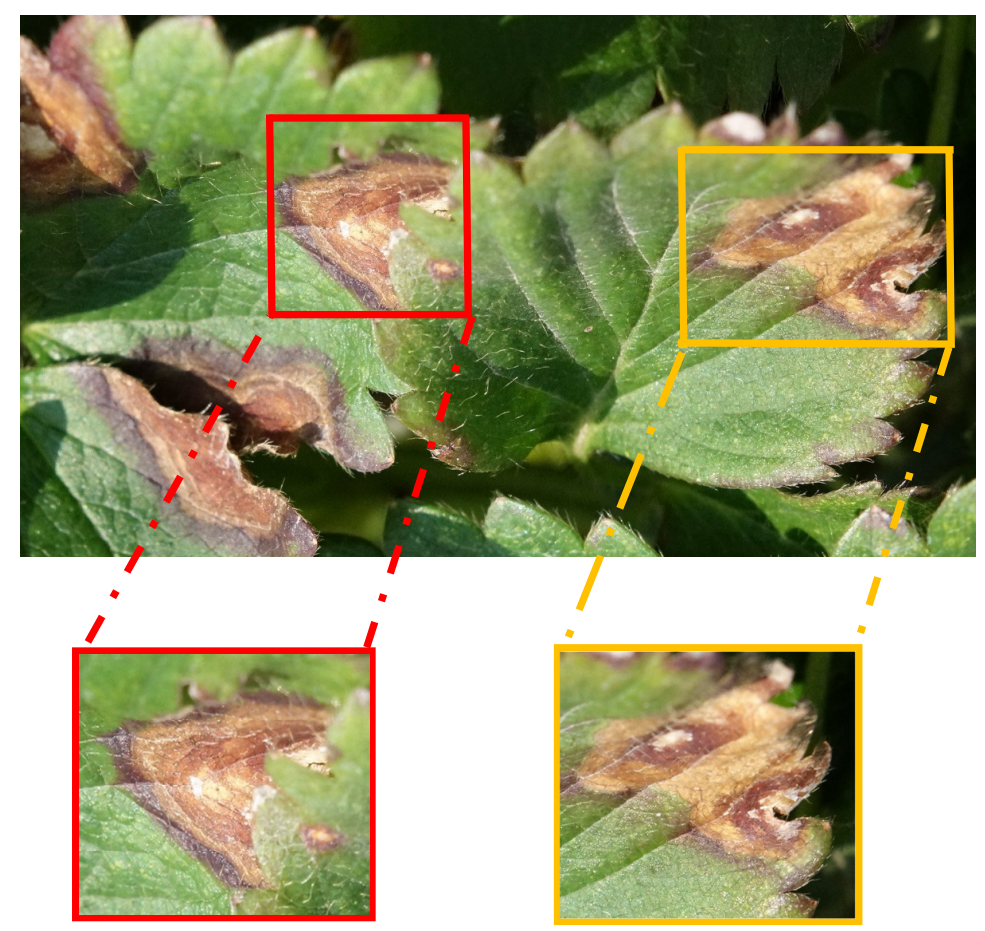

Figure 5. Schematic diagram of original image feature extraction. 
Each of the withered leaves was cut off as a feature image. The numbers of original images and feature images are shown in Table 1. Two hundred sixty-seven feature images were trimmed from 156 original images for crown rot caused by leaf blight disease, 262 feature images were trimmed from 166 original images for leaf blight diseases, 253 feature images were trimmed from 155 original images for fruit rot caused by leaf blight disease, 250 feature images were trimmed from 157 original images for gray mold diseases, and 273 feature images were trimmed from 158 original images for powdery mildew disease. A total of 1306 images were trimmed from original images and named as feature images (Figure 6). It is noted that the healthy dataset was not collected in this study. However, in the future, the healthy dataset will be used to enhance the impact of the proposed technique.

Table 1. Table of numbers of disease images from original and feature data sets.

\begin{tabular}{cccc}
\hline Diseases & Tissues & Original Images & Feature Images \\
\hline \multirow{2}{*}{ leaf blight } & crown & 156 & 267 \\
\cline { 2 - 4 } & leaf & 166 & 262 \\
\cline { 2 - 4 } & fruit & 155 & 254 \\
\hline gray mold & fruit & 157 & 250 \\
\hline powdery mildew & fruit & 158 & 273 \\
\hline Total & & 792 & 1306 \\
\hline
\end{tabular}
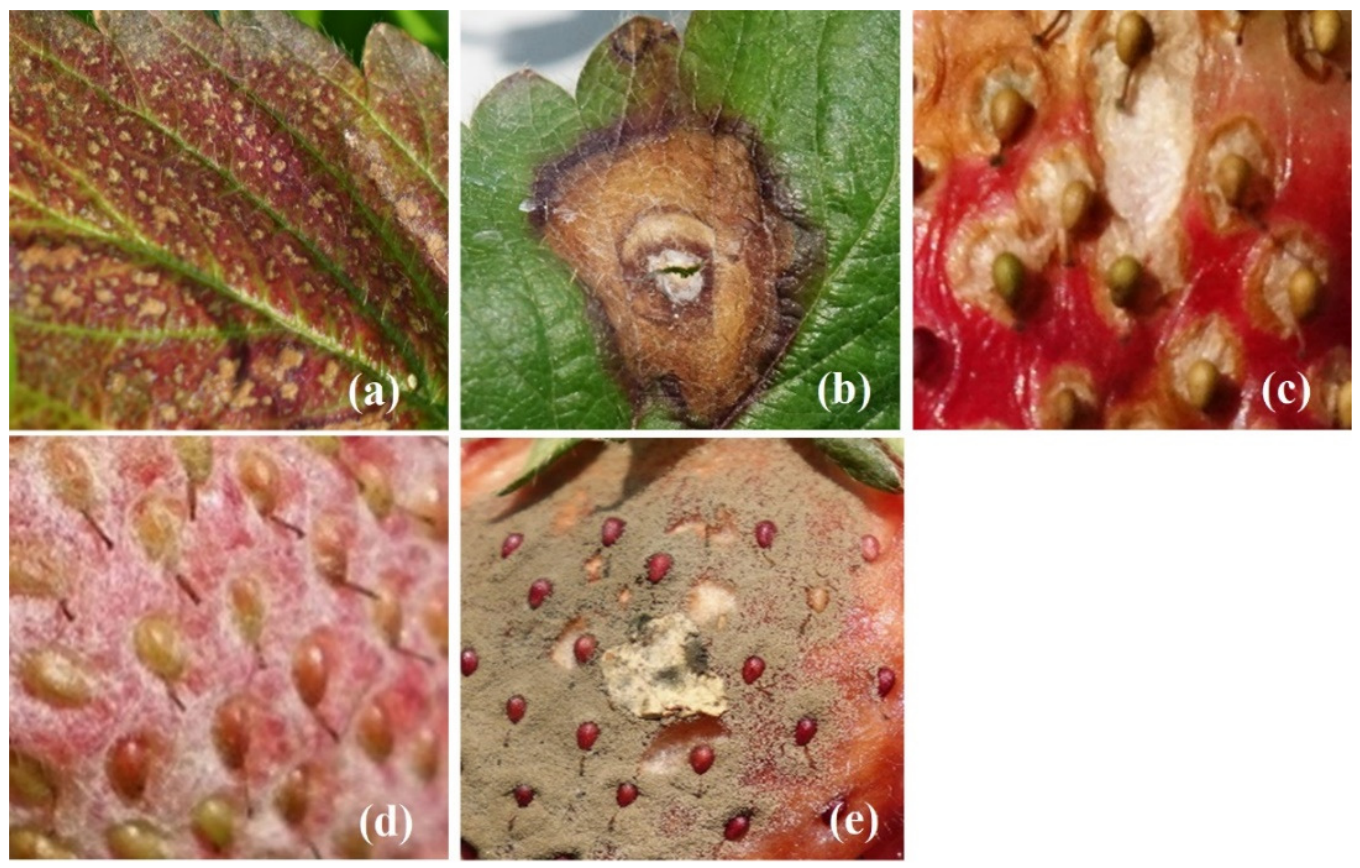

Figure 6. A total of 1306 feature images were trimmed from original images for different diseases of strawberry plant-(a) crown rot caused by leaf blight, (b) leaf blight, (c) fruit rot caused by leaf blight, (d) powdery mildew, and (e) gray mold.

\subsection{Convolution Neural Network Imaging Recognition}

\subsubsection{Convolution Neural Network Sketch}

$\mathrm{CNN}$ is a mathematical model that can simulate brain function and neural interactive connections based on a convolution process. In $\mathrm{CNN}$, the image was obtained from the input terminal and the image features are filtered out to the pooling layer through the convolution layer to sort out the new image features. The convolution and pooling are one layer. In the last layer, the final features are fully connected and identified according to the 
$\mathrm{CNN}$ algorithm. We used CNN to detect the different strawberry diseases based on the GoogLeNet, VGG16, Resnet50 algorithm. The sketch map of the CNN technique is shown in Figure 7.

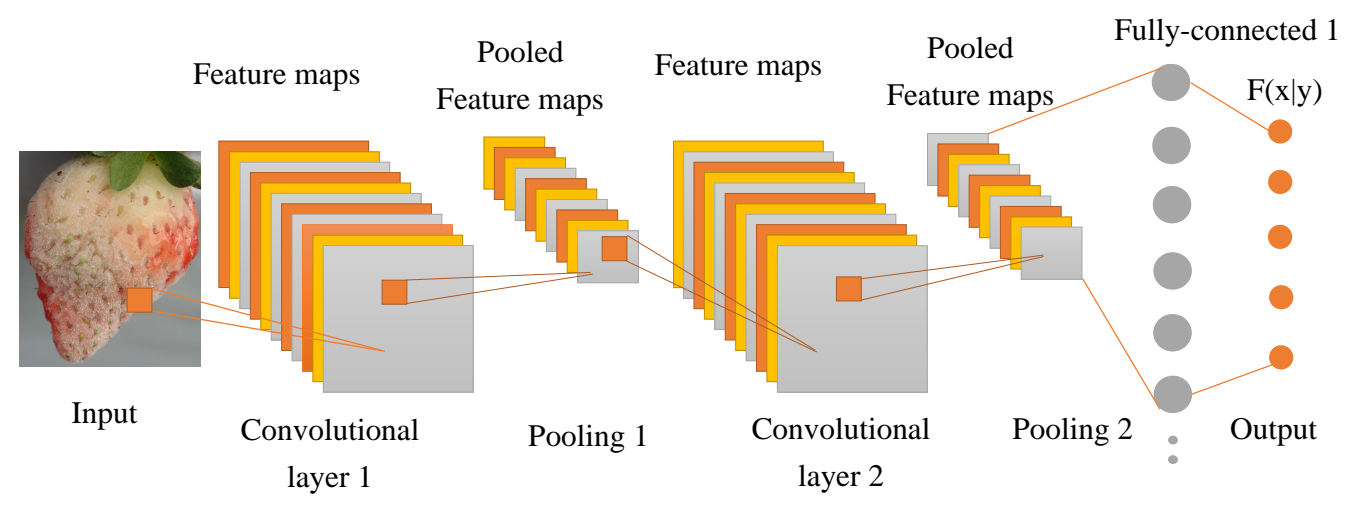

Figure 7. Convolution neural network for the detection of strawberry diseases.

Before inserting the input matrices into the neural network, the images were separated into the original and feature images. The original image dataset was used to instruct the neural network to focus on the neighborhood information and train it to extract necessary features through supervised backpropagation training. The background reduced from the feature image dataset was used to instruct the neural network to focus on the disease-like information and train the neural network to extract necessary features through supervised backpropagation training. For the output side, the neural network was trained like a pathologist making a diagnosis. This can be considered as an expert training method to convert human action into a computer algorithm.

\subsubsection{GoogLeNet Structure Diagram}

GoogLeNet, also known as Inception V1, won the championship in the 2014 imageNet image classification competition. The input image size was $224 \times 224$. The network uses a sparse structure (Figure 8). The structure uses $1 \times 1,3 \times 3,5 \times 5$ convolution kernels and a $3 \times 3$ pooling layer to perform parallel operations at the same time. After the operation is completed, all the convolved feature images will overlap to generate a new feature image.

\subsubsection{VGG16 Structure Diagram}

Vgg16 won the runner-up in the 2014 imageNet image classification competition. Vgg16 inherited the 2012 AlxeNet network design ideas and increased from eight to 16 layers on the AlexNet network. There are 13 convolutional layers (Conv), five pooling layers (Pooling), and three fully connected layers (gray parts) (Figure 9). [27]

\subsubsection{Resnet50 Structure Diagram}

The Resnet50 model won the 2015 ILSVRC image classification championship. The Resnet50 model has a residual network layer. To solve the problem of degradation during the calculation, the residual network has a better calculation result when the roll machine layer learns new features during feature input [28]. The Resnet50 model includes five convolution layers. For instance, in the orange frame, the middle $3 \times 3$ convolutional layer first reduces the calculation under a dimension reduction $1 \times 1$ convolutional layer and then restores it under another $1 \times 1$ convolutional layer. This not only maintains accuracy but also reduces the amount of calculation (Figure 10).

\subsubsection{CNN Traning}

The CNN model was coded using MATLAB. The model was run using an 80:20 ratio of 80:20 of training and testing data per training period (Figure 11). The training parameters are listed in Table 2 . When performing the training, Net can only process RGB images that 
are $224 \times 224$, used enhancement color processing, converting a grayscale image to RGB. To generate a new batch, images were input into the neural network, and weights were adjusted until the image features were analyzed. Data expansion techniques to increase the image by rotation and flips were used to overcome the overfitting problem when training with a small dataset [29]. We used the $\mathrm{CUAD}^{\circledR}$ the program to run the algorithm with the NVIDIA ${ }^{\circledR}$ RTX2070 GPU.

The average training accuracy and loss for the original and feature image datasets were calculated through three differential training periods of 20 epochs. The corresponding results of average training accuracy and loss rates were calculated for the original and feature image datasets (Figures 12 and 13). The training accuracy rates for VGG-16, GoogLeNet, and Resnet-50 were 97.42\%, 96.13\%, and 98.06\%, respectively (Figure 12). As shown in Figure 12, the training accuracy rates are $99.20 \%, 98.80 \%$, and $99.60 \%$, respectively, and VGG-16, GoogLeNet, and Resnet-50 correspond to each other. The Resnet50 model had the highest training accuracy results for the three training periods $(97.42 \%, 96.13 \%$, and $98.06 \%$ ). The training accuracy rates for the feature data set were $99.20 \%, 98.80 \%$, and $99.60 \%$ for each training period. The training accuracy rates increased with the increment of the training period of 5 to 10 epochs but subsequently decreased when the increment of the training period was changed from 15 epochs to 20 epochs. Furthermore, the accuracy of the feature image dataset was higher than that of the original image dataset. It can be explained due to the mix and complexity of the background patterns shown in the original image dataset. The system tends to be confused with several classes that results in lower performance.

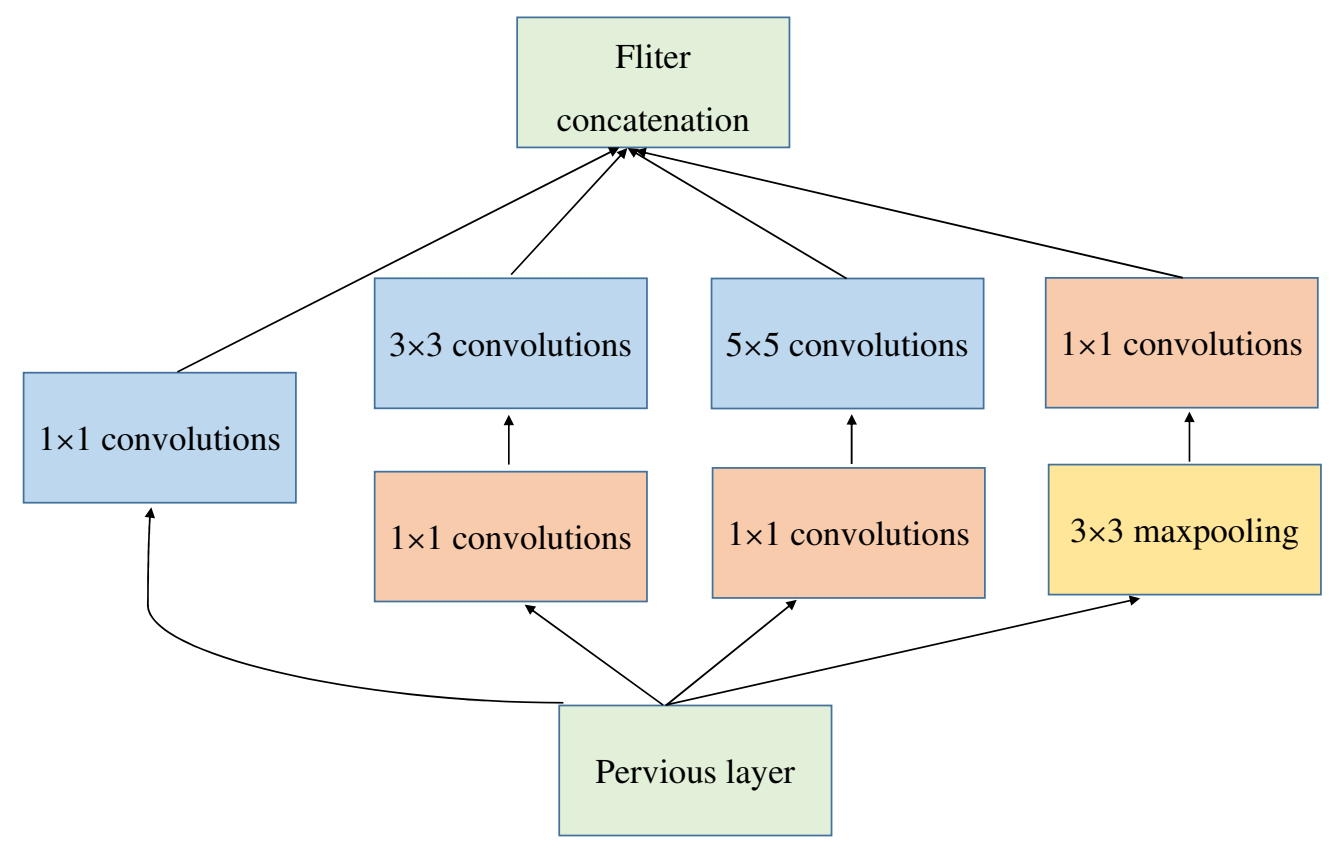

Figure 8. Diseases Inception Model structure diagram. 


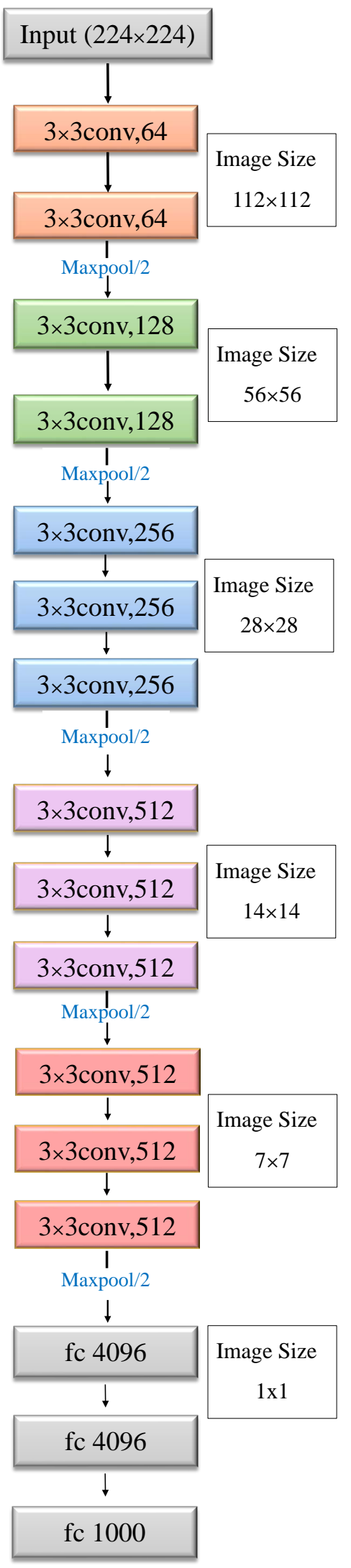

Figure 9. Vgg16 network architecture diagram. 


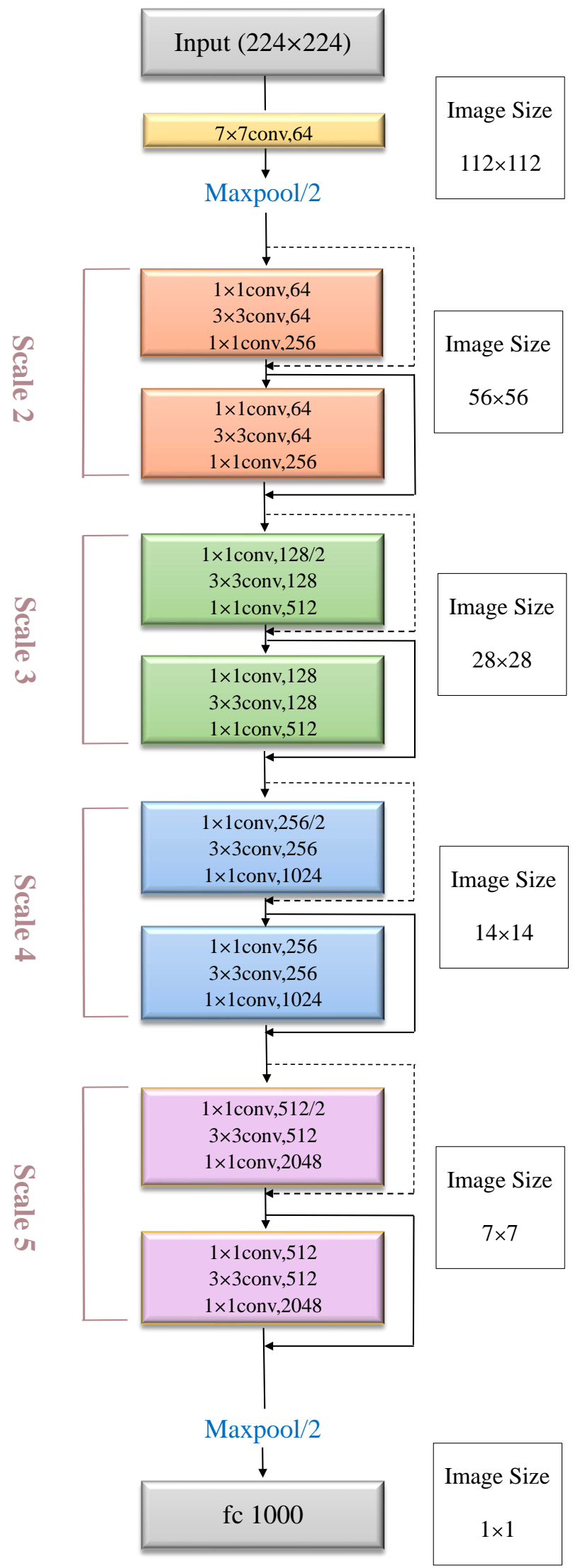

Figure 10. Resnet50 model for the detection of strawberry diseases. 


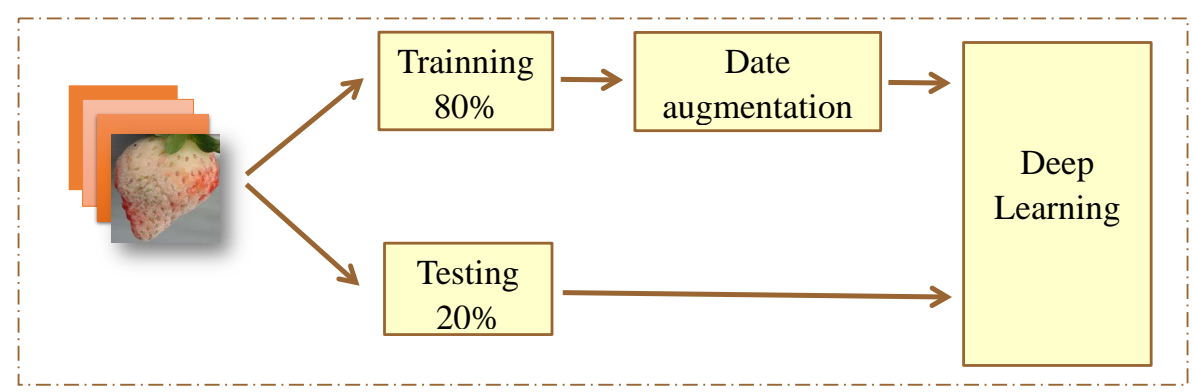

Figure 11. CNN training ratio distribution diagram for detection of strawberry diseases.

Table 2. Model training parameters.

\begin{tabular}{cc}
\hline Parameter Name & Value \\
\hline Optimization & sgd \\
Epochs & 20 \\
ValidationFrequency & 30 \\
Mini Batch size & 32 \\
Learning rate & 0.0001 \\
Execution environment & GPU \\
\hline
\end{tabular}

(a)

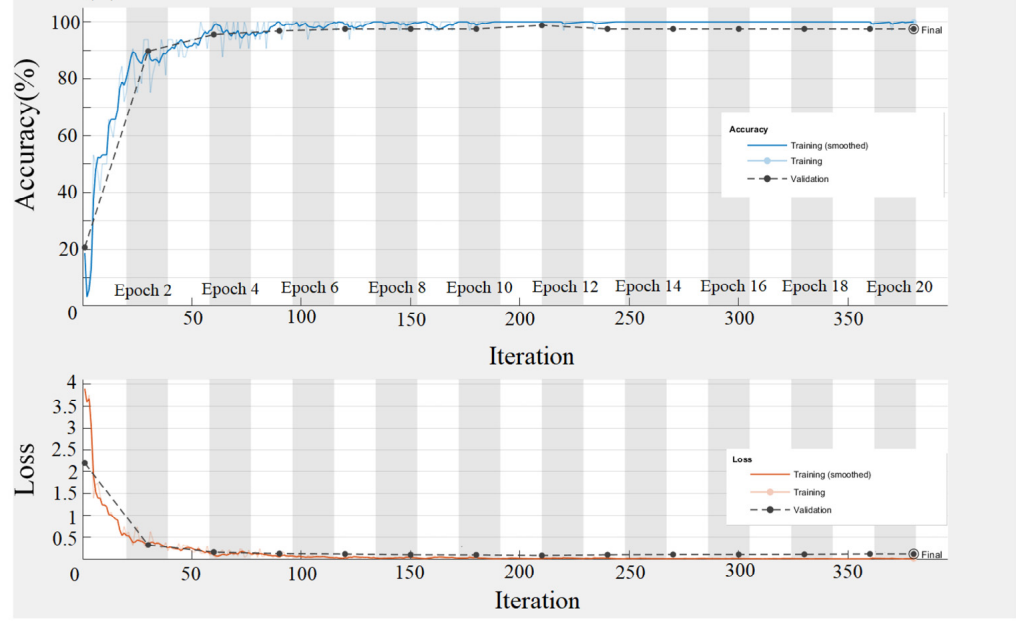

(b)
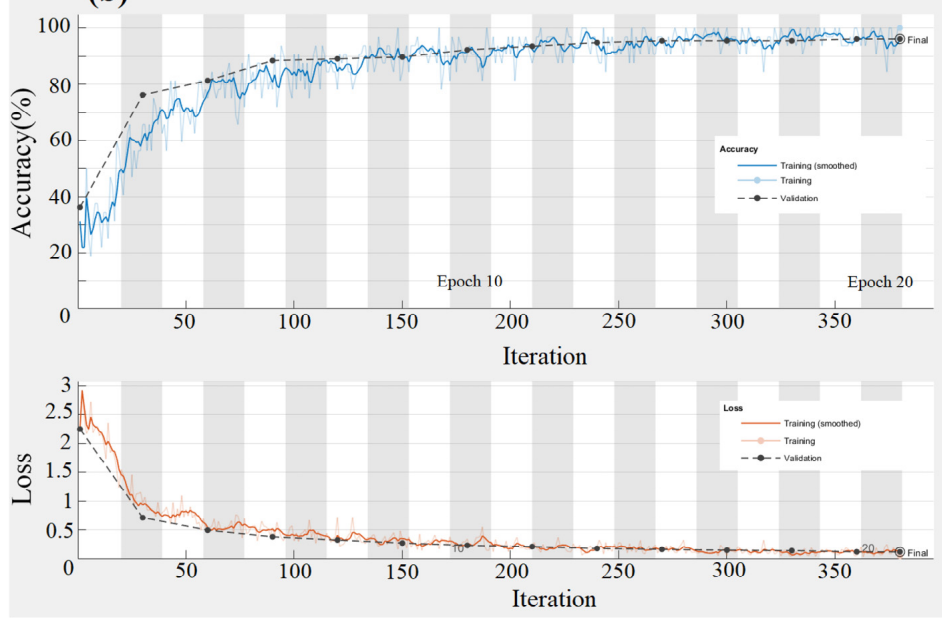

Figure 12. Cont. 


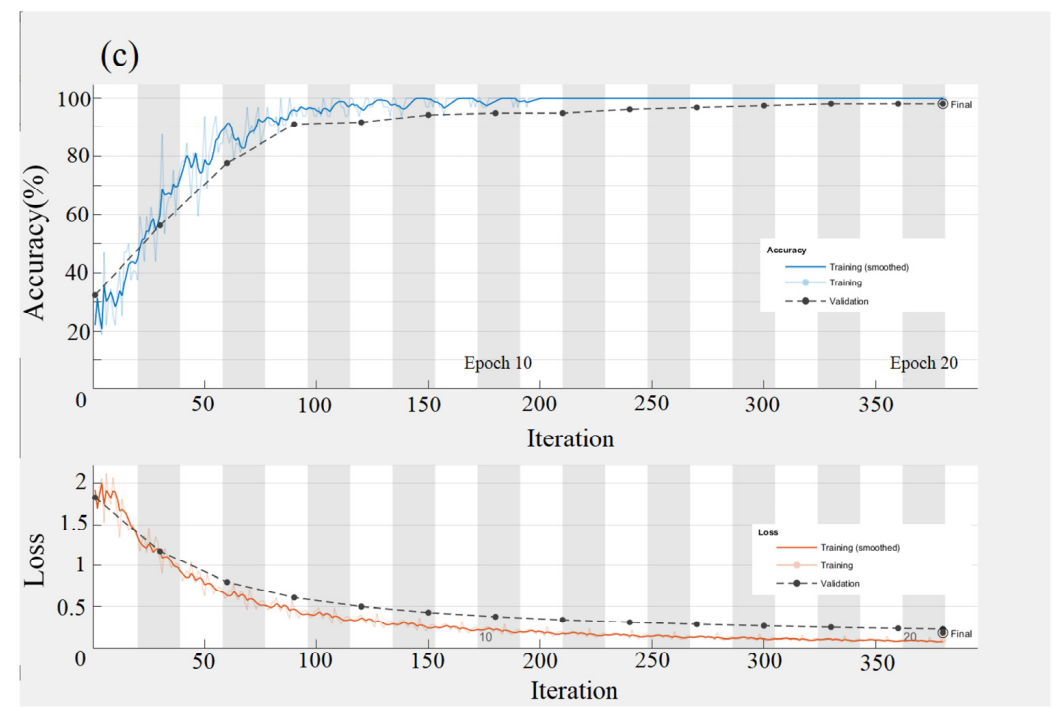

Figure 12. Accuracy and loss training map of original data set-(a) Vgg16, (b) GoogLeNet (c) Resnet50.

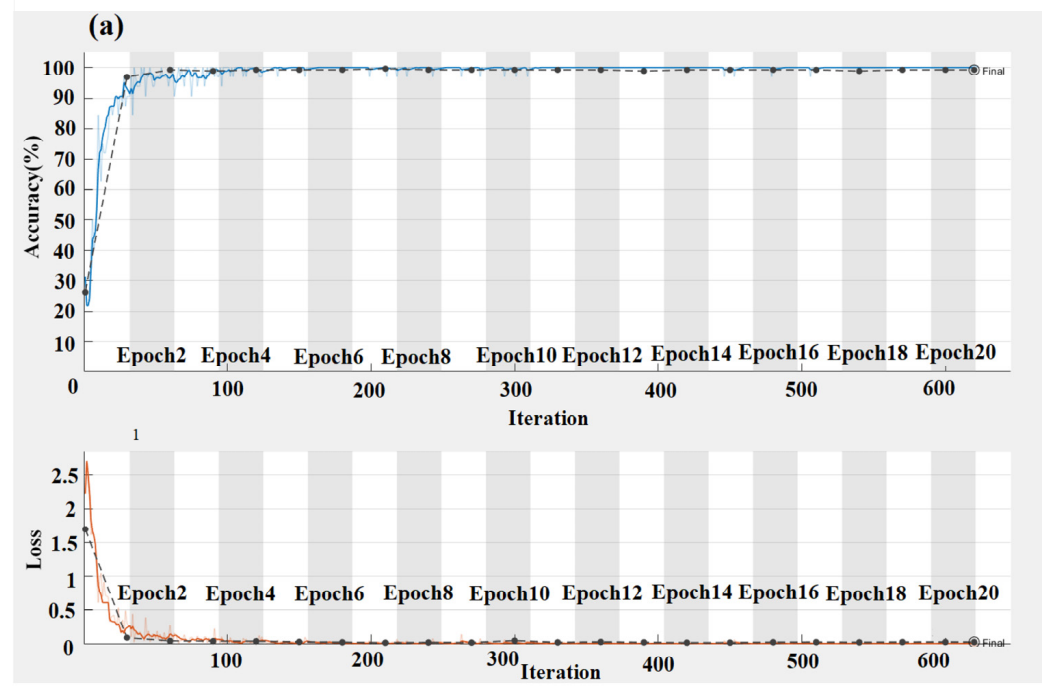

(b)
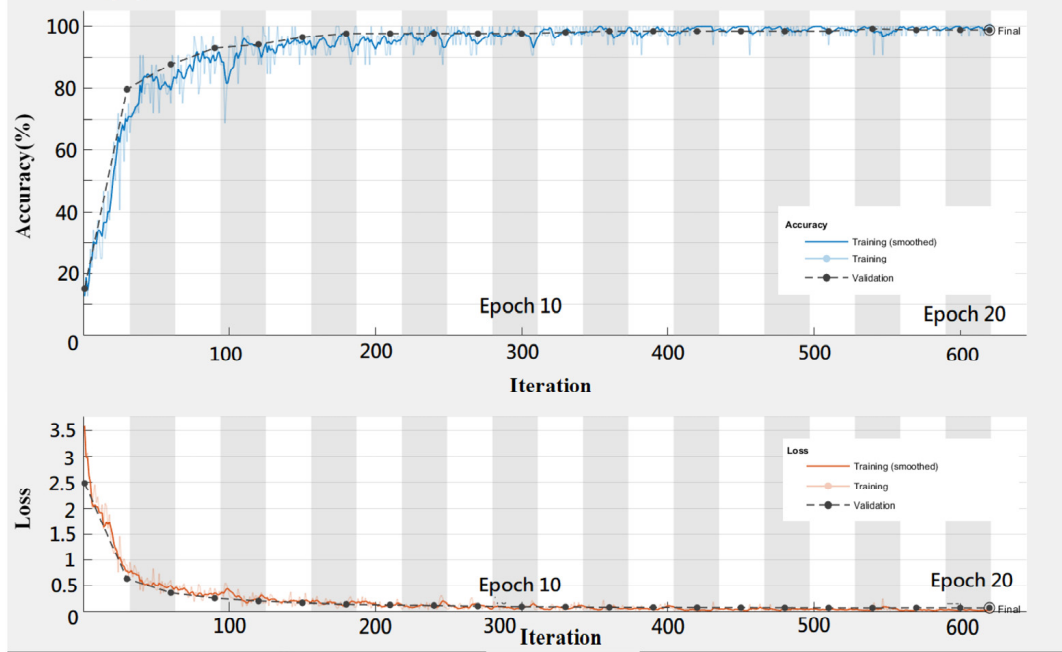

Figure 13. Cont. 


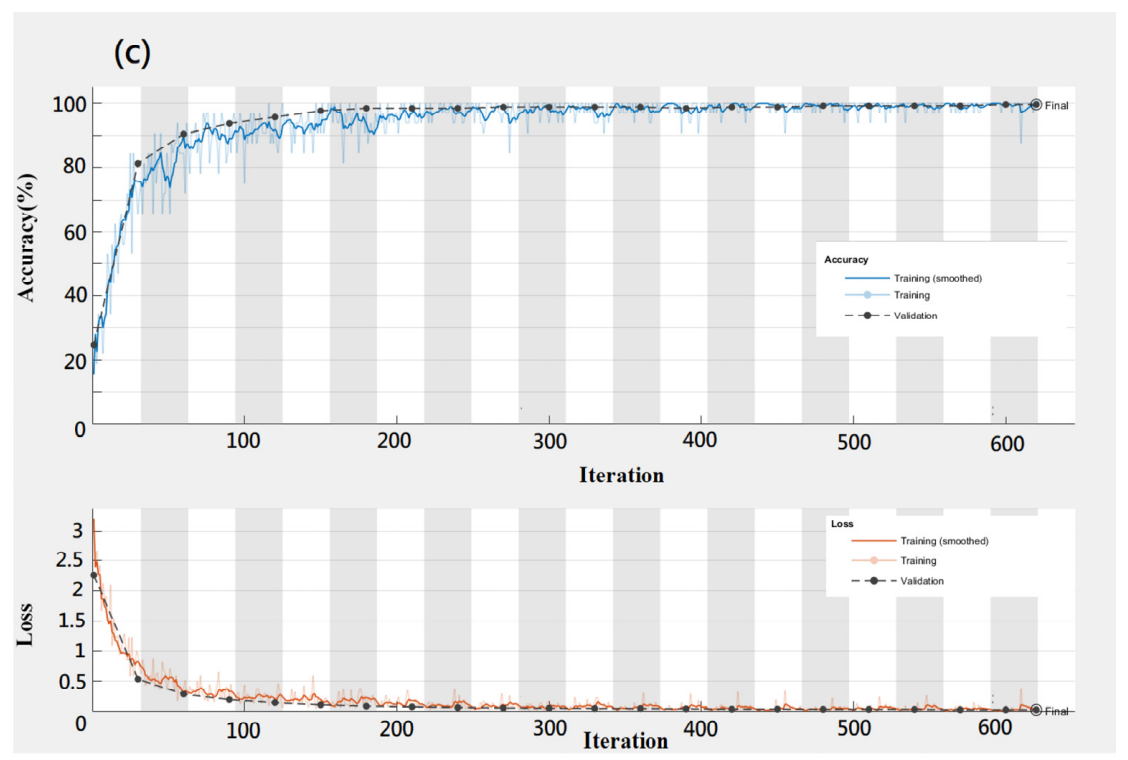

Figure 13. Accuracy and loss training map of feature data set-(a) VGG16, (b) GoogLeNet (c) Resnet50.

\section{Conclusions}

The CNN algorithm with the Resnet50 model is proposed for detecting strawberry diseases, namely crown leaf blight, leaf blight, fruit leaf blight, gray mold, and powdery mildew, using original and feature image datasets. The training accuracy was highest at the period of 20 epochs, with $98.06 \%$ and $99.60 \%$ accuracy for the original and feature datasets, respectively. The proposed approach provides a simple technique for detecting strawberry diseases, which will be important for agricultural use.

Author Contributions: Conceptualization, methodology, visualization and supervision M.T.-K.H.; software, formal analysis, data curation, writing-original draft preparation, J.-R.X.; investigation and resources, P.-C.C., H.-Y.W.; data curation, writing-review and editing, Q.-H.P.; supervision, project administration, and supervision, J.-L.A.Y.; All authors have read and agreed to the published version of the manuscript.

Funding: This research was funded by Ministry of Science and Technology of Taiwan, grant number MOST 108-2622-E-239-007-CC3 and MOST 107-2221-E-007 -110 -MY3.

Acknowledgments: The authors gratefully acknowledge the financial support provided to this study by the Ministry of Science and Technology of Taiwan (MOST) under Grant No. 108-2622-E-239-007-CC3 and MOST 107-2221-E-007-110-MY3.

Conflicts of Interest: The authors declare no conflict of interest.

\section{References}

1. Li, Y.F.; Wang, H.X.; Dang, L.M.; Niaraki, A.S.; Mon, H.J. Crop pest recognition in natural scenes using convolutional neural networks. Comput. Electron. Agric. 2020, 169, 105174. [CrossRef]

2. Skrovankova, S.; Sumczynski, D.; Mlcek, J.; Jurikova, T.; Sochor, J. Bioactive compounds and antioxidant activity in different types of berries. Int. J. Mol. Sci. 2015, 16, 24673-24706. [CrossRef] [PubMed]

3. Tylewicz, U.; Mannozzi, C.; Romani, S.; Castagnini, J.M.; Samborska, K.; Rocculi, P.; Rosa, M.D. Chemical and physicochemical properties of semi-dried organic strawberries. LWT 2019, 114, 108377. [CrossRef]

4. Pan, L.; Zhang, W.; Zhu, N.; Mao, S.; Tu, K. Early detection and classification of pathogenic fungal disease in post-harvest strawberry fruit by electronic nose and gas chromatography-mass spectrometry. Food Res. Int. 2014, 62, 162-168. [CrossRef]

5. Maas, J.L. Strawberry diseases and pests-progress and problems. In Proceedings of the VII International Strawberry Symposium 1049, Beijing, China, 18 December 2012; pp. 133-142.

6. Paulus, A.O. Fungal diseases of strawberry. HortScience 1990, 25, 885-889. [CrossRef]

7. Chung, P.C.; Wu, H.Y.; Ariyawansa, H.A.; Tzean, S.-S.; Chung, C.-L. First report of Anthracnose crown rot of strawberry caused by Colletotrichum siamense in Taiwan. Plant Dis. 2020, 103, 1775. [CrossRef] 
8. Chen, X.Y.; Dai, D.J.; Zhao, S.F.; Shen, Y.; Wang, H.D.; Zhang, C.Q. Genetic diversity of Colletotrichum spp. causing strawberry anthracnose in Zhejiang, China. Plant Dis. 2020, 104, 1351-1357. [CrossRef]

9. Feliziani, E.; Romanazzi, G. Postharvest decay of strawberry fruit: Etiology, epidemiology, and disease management. J. Berry Res. 2016, 6, 47-63. [CrossRef]

10. Petrasch, S.; Knapp, S.J.; Van Kan, J.A.; Blanco-Ulate, B. Grey mould of strawberry, a devastating disease caused by the ubiquitous necrotrophic fungal pathogen Botrytis cinerea. Mole. Plant Pathol. 2019, 20, 877-892. [CrossRef]

11. Chamorro, M.; Aguado, A.; De los Santos, B. First report of root and crown rot caused by Pestalotiopsis clavispora (Neopestalotiopsis clavispora) on strawberry in Spain. Plant Dis. 2016, 100, 1495. [CrossRef]

12. Amsalem, L.; Freeman, S.; Rav-David, D.; Nitzani, Y.; Sztejnberg, A.; Pertot, I.; Elad, Y. Effect of climatic factors on powdery mildew caused by Sphaerotheca macularis f. sp. fragariae on strawberry. Euro J. Plant Pathol. 2006, 114, 283-292. [CrossRef]

13. Rebollar-Alviter, A.; Silva-Rojas, H.V.; Fuentes-Aragón, D.; Acosta-Henández, U.; Martínez-Ruiz, M.; Parra-Robles, B.E. An emerging strawberry fungal disease associated with root rot, crown rot and leaf spot caused by Neopestalotiopsis rosae in Mexico. Plant Dis. 2020, 104, 2054-2059. [CrossRef] [PubMed]

14. Mahnud, M.S.; Zaman, Q.U.; Esau, T.J.; Price, G.Q.; Prithiviraj, B. Development of an artificial cloud lighting condition system using machine vision for strawberry mildew disease detection. Comput. Electron. Agric. 2019, 158, $219-225$.

15. Jayawardena, R.S.; Huang, J.K.; Jin, B.C.; Yan, J.Y.; Li, X.H.; Hyde, K.D.; Zhang, G.Z. An account of Colletotrichum species associated with strawberry anthracnose in China based on morphology and molecular data. Mycosphere 2016, 8, 1147-1163. [CrossRef]

16. Esgario, J.G.M.; Krohling, R.A.; Ventura, J.A. Deep learning for classification and severity estimation of coffee leaf biotic stress. Comput. Electron. Agric. 2020, 169, 105162. [CrossRef]

17. Lottes, P.; Behley, J.; Milioto, A.; Stachniss, C. Fully convolutional networks with sequential information for robust crop and weed detection in precision farming. Comput. Electron. Agric. 2018, 3, 2870-2877. [CrossRef]

18. Ferentinos, K.P. Deep learning models for plant disease detection and diagnosis. Comput. Electron. Agric. 2018, 145, 311-318. [CrossRef]

19. Cheng, X.; Zhang, Y.H.; Chen, Y.Q.; Wu, Y.Z.; Yue, Y. Pest identification via deep residual learning in complex background Comput. Electron. Agric. 2017, 141, 351-356. [CrossRef]

20. Selvaraj, M.G.; Vergara, A.; Ruiz, H.; Safari, N.; Elayabalan, S.; Ocimati, W.; Blomme, G. AI-power banana diseases and pest detection. Plant Methods. 2019, 15, 92. [CrossRef]

21. Mohanty, S.P.; Hughes, D.P.; Salathe, M. Using deep learning for image based plant disease detection. Front. Plant Sci. 2016, 7, 1419. [CrossRef]

22. Chen, Y.; Lee, W.S.; Gan, H.; Peres, N.; Fraisse, C.; Zhang, Y.C.; He, Y. Strawberry yield prediction based on a deep neural network using high resolution aerial orthoimages. Remote. Sens. 2019, 11, 1584. [CrossRef]

23. Sustika, R.; Subekti, A.; Pardede, H.F.; Suryawati, E.; Mahendra, O.; Yuwana, S. Evaluation of deep convolutional neural network architectures for strawberry quality inspection. Int. J. Eng. Tech. 2018, 7, 75-80.

24. Shin, J.; Chang, Y.K.; Heung, B.; Nguyen, T.Q.; Price, Q.W.; Almallhi, A. Effect of directional augmentation using supervised machine learning technologies: A case study of strawberry powdery mildew detection. Biosyst. Eng. 2020, 194, 49-60. [CrossRef]

25. Gao, Z.M.; Shao, Y.Y.; Xuan, G.T.; Wang, Y.X.; Liu, Y.; Han, X. Realtime hyperspectral imaging for the in-field estimation of strawberry ripeness with deep learning. Artif. Intell. Agri. 2020, 4, 31-38.

26. Nie, X.; Wang, L.; Ding, H.X.; Xu, M. Strawberry verticillium wilt detection network based on multi-task learning and attention. IEEE Access 2020, 7, 170004. [CrossRef]

27. Simonyan, K.; Zisserman, A. Very deep convolutional networks for large-scale image recognition. arXiv 2014, arXiv:1409.1556v6.

28. He, K.; Zhang, X.; Ren, S.; Sun, J. Deep residual learning for image recognition. In Proceedings of the IEEE Conference on Computer Vision and Pattern Recognition, Las Vegas, NV, USA, 27-30 June 2016; pp. 770-778.

29. Montserrat, D.M.; Lin, Q.; Allebach, J.; Delp, E.J. Training object detection and recognition CNN models using data augmentation. Symp. Electron. Imaging 2017, 2017, 27-36. [CrossRef] 